Revue d'histoire de l'Amérique française

ZBS REVUE D.HISTOIRE DE L'AMÉRIQUE FRANÇAISE

\title{
Un texte de La Galissonière sur les Antilles françaises
}

\section{Roland Lamontagne}

Volume 19, numéro 4, mars 1966

URI : https://id.erudit.org/iderudit/302516ar

DOI : https://doi.org/10.7202/302516ar

Aller au sommaire du numéro

Éditeur(s)

Institut d'histoire de l'Amérique française

ISSN

0035-2357 (imprimé)

1492-1383 (numérique)

Découvrir la revue

Citer ce document

Lamontagne, R. (1966). Un texte de La Galissonière sur les Antilles françaises. Revue d'histoire de l'Amérique française, 19(4), 606-625.

https://doi.org/10.7202/302516ar d'utilisation que vous pouvez consulter en ligne.

https://apropos.erudit.org/fr/usagers/politique-dutilisation/ 


\section{DOCUMENTS INEEDITS}

\section{UN TEXTE DE LA GALISSONIËRE SUR LES ANTILLES FRANÇAISES}

Démuni de date et de signature, ce document ${ }^{1}$ ne révèle pas la connaissance grammaticale qui caractérise les écrits autographes de Barrin de La Galissonière. Il est possible qu'il ait été rédigé sous la dictée. Il comporte notamment une erreur toponymique; on lit Cap Anne Marie alors que les cartes indiquent Cap Dame Marie.

Ce texte rassemble des considérations d'ordre militaire touchant les établissements portuaires de la Martinique et de Saint-Domingue ${ }^{2}$. Il met en lumière l'interdépendance de la marine, du commerce et des colonies de l'Amérique française à un moment critique de faiblesse maritime de la France.

Aucun passage n'est souligné dans la copie originale. La présentation en italique a pour but d'éclairer l'interaction: marine - commerce - colonies. Dans la première moitié du $\mathrm{XVIII}^{\mathrm{e}}$ siècle, une bourgeoisie libérale impose à la GrandeBretagne une politique d'expansion économique. L'équivalence que William Pitt établissait entre la politique et le commerce

1 Archives publiques du Canada. Bibliothèque Nationale de Paris, Fonds français, vol. 11340, fos 3-54. Ce texte intitulé brièvement Mémoire et conservé dans le Recueil de Mémoires concernant la marine de France figure à l'inventaire sous le titre suivant: Memoire contre les Projets des Anglois, sur nos Colonies Meridionales de l'Amérique, par $M^{r}$ de la Galissonniere (sic). La signature autographe de La Galissonière n'a qu'un n.

2 Voir Description topographique, physique, civile, politique et historique de la partie française de l'isle de Saint-Domingue, éditée à Philadelphie par Moreau de Saint-Méry et rééditée par Blanche Maurel et Etienne Taillemite (3 vol., Paris, 1958). - G. Debien, "Etudes antillaises au XVIII' siècle", Cahiers des Annales. Economies-Sociétés-Civilisations, No 11.

Conservée au Département de Géographie de l'Université de Montréal dans un atlas réédité vers 1800 (la page frontispice manque), une carte de Robert de Vaugondy illustre la "Partie de la Mer du Nord où se trouvent les Grandes et Petites Isles Antilles, et les Isles Lucayes, par le $S^{r}$ Robert". Gilles Robert de Vaugondy, 1688-1766, cartographe et géographe du roi, a publié des atlas en 1748, 1758 et 1762. 
Hoventre 1) 46.

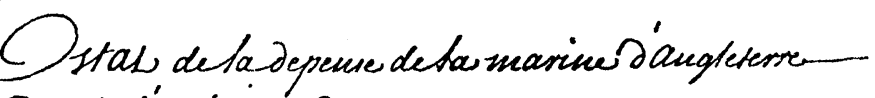
Depuis tániée ij36.

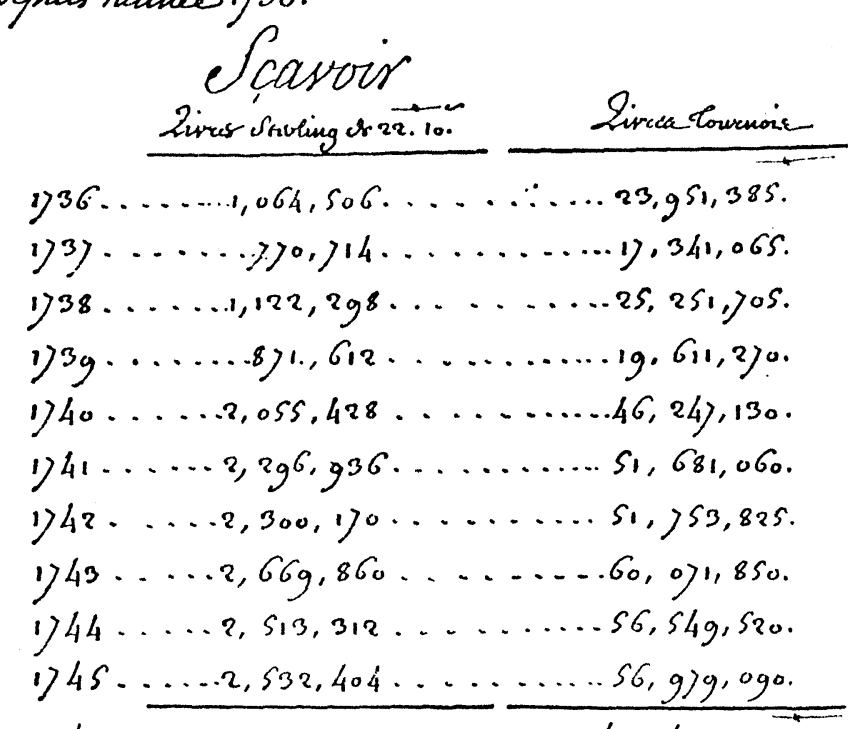

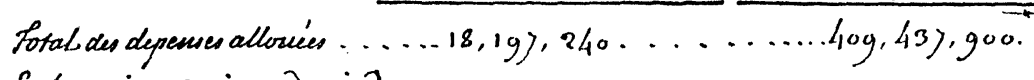

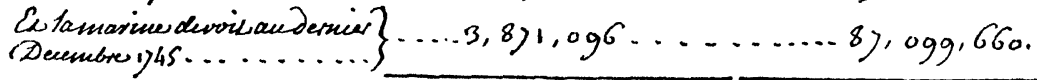

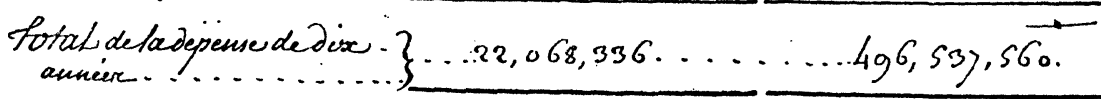

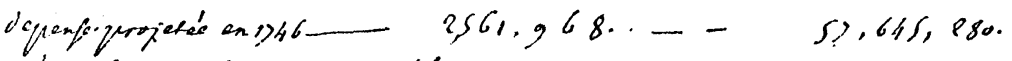

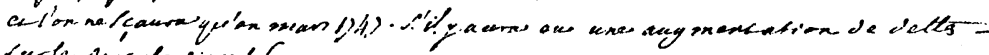
Surles oppeypousgh6.

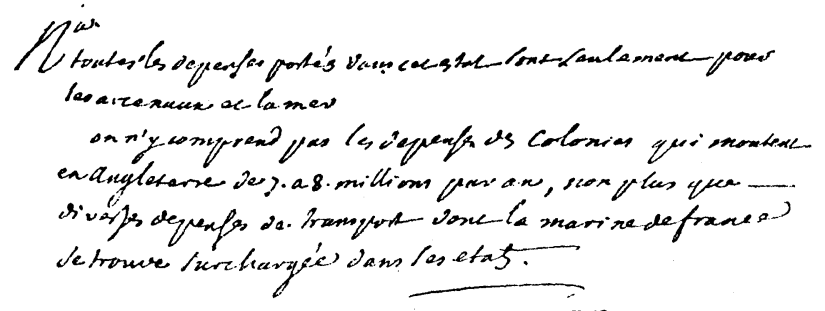

Maurepas Papers, Lot no 48. Cornell University Library.

[607] 
caractérise les préoccupations des autorités anglaises noyautées autour de financiers, d'armateurs et de négociants. La Galissonière rappelle l'ambition de conquête coloniale dans la Mer des Antilles, de la part de William Pitt, au début de la Guerre d'Amérique.

Maurepas a pris conscience du fait que la Grande-Bretagne a apporté un soutien notable aux Anglo-Américains, au cours de la période de 1736 à 1745, pour n'indiquer que les années qui font l'objet d'une vérification à partir d'une source manuscrite. En marge de cet "Estat de la depense de la marine d'Angleterre depuis l'année 1736", il note: “on n'y comprend pas les depenses des Colonies qui montent en Angleterre de 7 a 8 millions par an, non plus que diverses depenses de transport dont la marine de france se trouve surchargée dans ses etats." 3 Si l'on se réfère au bilan, cette somme était probablement exprimée en livres sterling.

Vers cette époque, les marchands français dominaient ies échanges économiques de Cadix qui avait remplacé Séville comme centre espagnol de relations commerciales avec l'Amérique. Ils épousaient des Espagnoles et s'implantaient dans la péninsule ibérique. Des sources administratives mentionnent que la Cour française devrait accorder aux marchands le prestige social et également encourager la haute noblesse à participer à la vie économique. ${ }^{4}$

Ce document de Barrin de La Galissonière éclaire un aspect de “cette Méditerranée, selon Pierre Deffontaines, qu'est la Mer des Antilles autour de laquelle s'établissent, comme dans l'Ancien Monde, les grandes civilisations continentales, Amérique du Nord, du Sud et Centrale". 5 Il s'insère dans cette dynamique d'ensemble que constitue l'étude des océans et des mers, ces grandes aires de l'expansion coloniale. ${ }^{6}$

Université de Montréal

ROLAND LAMONTAGNE

3 Maurepas Papers, Lot No 48. Cornell University Library.

4 Catalogue No 2092 (Parke-Bernet Galleries, New York, 1962). Répertoire analytique des papiers de Maurepas, voir pages 33, 96, 98.

5 "Condition de l'homme", La Nef, Cahier No 13 (Juin 1956) : 138. 292.

6 Frédéric Mauro, L’Expansion Européenne, 1600-1870 (Paris, 1964), 


\section{MEMOIRE 1}

On ne peut plus se dissimuler le danger évident et prochain que courent les Colonies françoises de l'Amérique méridionale, depuis que la Marine Royale paroist hors d'etat de les deffendre ouvertement et la marine marchande de les munir et de les approvisionner abandonnées a elles mesmes pendant la paix, la situation des choses dans la guerre presente les expose a toutes les entreprises des ennemis.

St-Domingue qui devoit être lobjet le plus considerable de l'attention de la France, sera naturellement aussy que tentera le plus les Anglois, et par cette raison et par la facilité du succès. Il est inconcevable qu'une colonie de cette importance dans les Ports de laquelle plus de 350 vaisseaux marchands abordent tous les ans, chargés de denrées superflues du Royaume, et qui raportent en retour celles du crû du pays qui forment au moins au total un article de 150 millions, ait été si mal connue jusqu'à présent, ou du moins si fort négligée excepté les négociants des places maritimes, il y a aussy peu de françois instruits de la valeur réelle de St-Domingue, qu'il y en a eu peu qui n'ayent exagéré celle de Louisbourg. On a une si foible idée de ce royaume, car c'est ainsy que l'on doit nommer une isle de plus de 750 lieues de tour, dont la partie fertile et abondante en ports apartient à la France, qu'il est assez ordinaire que lon confonde St-Domingue avec la Martinique le peu de connoissance sur cela est poussé si loin que l'on a lieu de croire que la perte de cette colonie eut fait moins de sensation dans le public que celle de l'Isle Royale.

Par malheur il a semblé jusqua present que ceux qui etoient fait pour estre plus éclairés et a qui le sort de toutes les colonies étoit confié, eussent adopté la meme opinion ou plustot la meme erreur. Sans cela eussent-ils laissé en proye aux ennemys de la France, la plus riche et la plus féconde branche, ou pour mieux dire, le véritable tronc de son commerce?

S'il a été facile aux Anglois de s'emparer de l'Isle Royale, il n'est pas vray qu'il leur est encore plus aisé de se rendre maîtres de St-Domingue, ou du moins d'y faire des ravages, qui équivaudroient pour longtems a sa perte. Les places sont depourvues de munitions de guerre et de bouche, de garnison

1 Bibliothèque Nationale, Fonds Français, vol. 11340, fos 1-67, Archives Publiques du Canada. - Voir les textes de Pierre Bouguer sur la Martinique et Saint-Domingue, cités par Roland Lamontagne, La Vie et 'Oeuvre de Pierre Bouguer (Paris, 1964), 24-29. 
suffisantes ${ }^{2}$, et fortifications susceptibles de resistances; et l'entrée de ses ports de deffenses capables d'arrêter la plus foible escadre. Ce n'est pas cependant qu'il n'ait été fait des depenses exorbitantes pour tous ces objets, mais l'employ en a été aussy infidèle que dans les autres colonies tout ce que la Métropole y a gagné, c'est de n'avoir rien fourny a la cupidité des administrateurs de ce pays qui s'entretient, paye ses troupes et ses fortifications tandis qu'elle s'est epuisee pour la conservation de ses etablissemens dans l'Amerique Septentrionale. Mais pour St-Domingue dont la sureté importe tant a la France, les suites peuvent n'en pas estre moins funestes, et le seroient beaucoup plus en effet, par la difference prodigieuse des interets.

Il seroit trop long sans doute de rappeler ici toutes les sources de malversations qui ont amené les choses au point ou elles sont aujourd'huy. Le detail n'en seroit necessaire qu'autant qu'elles ne seroient pas reconnues, et qu'un premier commis, despotique dans l'administration des colonies, le gouverneroit, encore impunément avec la dureté et les excès qu'il a exercés si iongtems. Iì en avoit fait son patrimoine et î̉ n'en confioit la direction qua des mains devouées a ses vues Labus de cette autorité a cause le mal que l'on expose icy. Il est si grand et les circonstances sont si peu favorables, qu'il est difficile d'y apporter des remedes bien efficaces cependant il faut le tenter, après avoir examiné tant les moyens de supléer a la foiblesse actuelle des Places que ceux de les munir de troupes, de vivres et de poudre.

Le Port Dauphin est le premier Etablissement de la cote du nord de St-Domingue. Son entrée est si étroite, qu'un vaisseau n'y peut eviter et qu'il est obligé de passer a la petite portée du pistolet du fort de la Bouque, qui est situé sur la pointe gauche. La difficulté de ce passage est cause que les vaisseaux du Roy ne vont presque jamais mouiller dans la Rade du fort Dauphin; et qu'il y a très peu de pilotes, pratiqués du pays, en état de les y entrer. La meme raison ne le rend pas d'un accès moins difficile aux Ennemis. Et il leur seroit en effet impraticable; si le fort de la Bouque étoit construit de façon à résister a quelques vaisseaux embossés. Mais il presente une Ligne de feu si peu étendue, son canon est tellement hors d'Etat de servir faute de recul, et en tout il est si foible, que l'on ne doit pas le considerer comme un boulevard sur lequel il puisse se reposer de la deffense du fort Dauphin; D'ailleurs rien de plus aisé que de le tourner par terre dou il est accessible de tous

2 sic. 
cotés. Le fort Dauphin destiné a battre l'intérieur de la Rade n'est pas meilleur. C'est un amas de pierres mal assemblées et du Canon duquel il est fort $^{3}$ de se mettre à l'abry, derriere une petite isle qui est à droite Et qui n'a aucune fortification, quoique de la on puisse bombarder facilement le fort Dauphin. Le Débarquement y est tout aussi praticable en plusieurs endroits; et l'on ne peut les garantir que par des retranchemens, mais il faut qu'ils soient gardés par des troupes suffisantes faute d'en avoir assé de reglées pour garnir tous les postes de cette grande colonie, comme on le verra, il est necessaire d'employer au fort Dauphin, ainsy qu'ailleurs, outre les milices et les Negres, esclaves choisis parmi les meilleurs des habitations qui sont de la dependance du fort Dauphin en exerçant ces nègres au service du canon, et a charger et tirer un fusil, on suppléera a ce qui manque pour bien garder les retranchemens. Il seroit juste de faire envisager des recompenses a ceux qui se distingueroient et de payer leur valeur a leurs maistres, soit qu'ils fussent affranchis pour quelque action, soit qu'ils fussent tués ou estropiés par les ennemis, mais il faudroit que cette valeur fut le prix réel du nègre et non 600 \# à quoy lon a taxé ceux que la justice condamne à la mort, la modicité de cette somme empesche souvent les propriétaires de lui dénoncer des nègres d'un grand prix et de la l'Impunité des crimes.

D'ailleurs il faut tant de formalités pour obtenir le payment de ces 600 \# que l'on aime mieux les abandonner, la caisse sur laquelle ces derniers sont pris se nomme la caisse des supliciés cet impot se lève comme la capitation en taxant chaque nègre. La taxe est de quarante sols il $\mathrm{y}$ a environ 150,000 nègres dans la colonie, ainsy elle monte par chaque année à $300,000 \#$. Et souvent il n'en sort pas un sols, pour l'objet qui a donné lieu a cette imposition: l'employ de l'argent qu'il produit est secret c'est l'Intendant qui le tire des mains du Receveur et l'on ignore a qui il rend compte. Quoi qu'il en soit on voit par ce detail que la caisse des Supliciés est plus que suffisante pour payer les nègres employés a la deffense de leurs quartiers comme on le propose. C'est le moyen le plus efficace et le plus prompt a employer pour supléer a ce qui manque des troupes à St-Domingue.

LE CAP FRANCAIS est le 2e port qui se présente également au nord, et au Vent de l'Isle, et le premier par son importance, et sa supériorité sur tous les autres. Voicy son état présent, on proposera ensuite ce que lon pense de plus convenable pour le rendre moins critique. Il y a deux entrées, pour arriver dans

$\mathbf{s}$ sic. 
la rade du cap, connus sous le nom de Grandes et de Petite passes. La grande est sur la gauche entre deux ecueils appelés la coque vieille, et le mouton. La petite est adroite entre le mouton et la pointe de picolet. Cette dernière est la plus étroite et la moins fréquentée mais elle est cependant très praticable, plus courte que la première et mieux gardée, ou du moins plus facile à garder comme on le prouvera. La grande passe est celle que prennent presque tous les vaisseaux; et il est démontré pour qui connoit le local que le fort de picolet est un facile obstacle à vaincre pour y penetrer. Sa batterie inferieure est si peu etendue, que son feu en devient meprisable quand a sa batterie superieure elle est tellement elevée quelle ne peut meme atteindre les vaisseaux dans la grande passe elle na été faite quen contregarde, pour battre la première, dans le cas ou les ennemis sen seroient emparés, d'ailleurs quand celle cy seroit aussy redoutable quelle est foible et mal construite les vaisseaux passent sy rapidement devant elle qu'une Escadre a le tems d'être garentie de son feu, avant que ses canons soient rechargés pour luy tirer une seconde volée.

C'est toujours avec un vent frais et régulier depuis 10: heures du matin jusqua cinq à six heures du soir, que lon entre dans la rade du cap: il est bien extraordinaire qu'avec cette connoissance on se soit borné à fortifier un point unique, pour en deffendre l'accès. Ce point est ce que l'on nomme le fort de Picolet. Il est adossé à une montagne qui le domine de plus de 400 toises de hauteur perpendiculaire en le prenant à revert, ce qui est la chose de monde la plus aisée. On sent qu'avec des pierres seulement que l'on y feroit rouler la place ne seroit point tenable, elle ne l'est pas meme au feu du canon dont les Boulets porteroient dans le roc que l'on a coupé, pour asseoir ce fort. On a si peu aplani le terrein, qu'il n'y a de profondeur que celle qu'il faut pour le recul du canon. Et au surplus la maçonnerie de cet ouvrage, comme de tous ceux qui se font dans nos colonies, est si mauvaise qu'il seroit a [c] raindre quelle n'éboulat au seul ébranlement de son artillerie. Telle est pourtant la forteresse qui est destinée a asseurer l'entrée de la rade du cap, c'est a dire du lieu de la plus grande importance que le Roy ait conserver encore cette forteresse peut-elle contenir a peine le nombre de canoniers qu'il luy faut, sans avoir de cazemates, de citerne, ny de lieu propre à placer des vivres: ainsy il en faut relever la garnison presque tous les jours en sorte que si les ennemis étoient maistres de la rade, ils empecheroient facilement la communication du cap à Picolet, et le prendroient a discretion pour se retirer s'ils estoient contraints sans en estre incommodés, le 
seul moment néantmoins ou il peut les combattre par la hauteur avec laquelle on sort du cap, d'ou il faut souvent se faire remarquer jusqua ce fort mais il n'y auroit meme pas a esperer qu'arrivés dans la rade, les ennemis fussent obligés de l'abandonner sans avoir ruiné la ville, par le peu de difficulté qu'ils y trouveroient. Toute sa deffense consiste en une batterie a l'extremité du quay sur le bord de la mer, qui n'est pas achevée, et qui est si écrasée qu'elle seroit dominée meme par les batteries basses des vaisseaux qui en auroient d'ailleurs aussitôt eteint le feu, qu'il leur seroit facile de le mettre au cap si leur expedition se bornoit a ce seul succes et qu'ils neussent point de trouppes de débarquement. Au reste toutes les plages qui y sont propres sont ouvertes tant dans l'Intérieur de la rade qu'aux environs; et le chemin de la terre leur est aussy libre que celuy de la mer, pour remedier momentanement à tant de cotés foibles, voicy ce que l'on pense qu'il conviendroit d'y faire a la hâte.

$1^{\circ}$ Couler a fond des vaisseaux dans la grande passe, $\mathrm{y}$ retablir des Brulots, et ne se reserver que la petite.

$2^{\circ}$ faire des retranchemens depuis Picolet jusquau Cap et les garnir de gros canons et de mortiers pour battre depuis son entrée jusqu'à son arrivée une escadre qui auroit des desseins sur cette ville.

$3^{\circ}$ En placer également sur l'Isle du carenage.

$4^{\circ}$ Achever la batterie du quai devant le Cap par la prolongation qui est projetée depuis longtems ainsy que lorillon en avant de la maison du Gouverneur, qui fournira le seul feu qui puisse être dirigé dans la grande passe; presque tous les canons de la Batterie du quai ne portant qu'au fond de la rade et ceux de la Batterie Circulaire vis a vis les anciennes cazernes ne se dirigeant guerre ${ }^{4}$ que dans la petite passe.

$5^{\circ}$ Il n'est pas moins important de fortifier et de garder ce défilé de la bande du Nord, par ou l'on peut être pris à revers, après un débarquement auquel rien ne s'oppose a présent pour cela 4 à 500 hommes suffiront, avec une douzaine de petits canons placés a propos pour arrêter des forces considerables dans des chemins etroits, difficiles et ou l'on peut faire promptement des coupures et des abbatis d'arbres si l'on se tient exactement sur ses gardes et que l'on ait toujours des travailleurs tout prêts.

4 sic. 
$6^{\circ}$ Il est nécessaire d'ajouter a la deffense de ces lieux importants des nègres esclaves, comme on la dit au sujet du fort Dauphin.

$7^{\circ}$ Il ne l'est pas moins de fermer le Cap, au moins en continuant ou en relevant les fortifications en terre que M. de Larnage avoit fait faire depuis la montagne jusqu'à la mer du costé de la plaine.

$8^{\circ}$ Enfin le meilleur expedient et peut être le seul qui puisse le mettre aujourdhuy en sureté et d'envoyer une escadre toujours prête a s'enfermer dans sa Rade, n'y eut il meme que deux ou trois vaisseaux de ligne placés de facon à diriger leur feu dans la petite passe, ils empescheroient certainement les ennemis de la forcer parce que le 1er de leur vaisseaux ${ }^{5}$, qui seroit desemparé exposeroit tous les autres a se perdre, ou sur les écueils de la droite, ou sur la cote.

Si au lieu de batir le Cap au pied d'une montagne enorme ou il ne peut jamais former une place de conséquence qui exige uni siège on lêut plâcé à la petite anis̃e, noun seủleminent lâ Raủe se seroit trouvée deffendue par des fortifications, mais son mouillage n'eut pas été tenable sous ce feu et il eut fallu un débarquement de 25 milles hommes pour l'attaquer régulièrement. On en sent facilement l'impossibilité par les frais immenses d'une pareille entreprise à 1800 lieues dou l'on part; et d'ailleurs le climat se fut toujours opposé au succès d'une tentative de longue haleine. Le Port de Paix vient après le Cap; c'est un endroit beaucoup moins considerable et moins essentiel mais aussy d'une deffense beaucoup plus facile, parce que la Plage accessible au débarquement est assez resserrée, et qu'elle ne sera point abordable quand les forts qui la protegent seront en bon état, et que leur artillerie sera bien servie. Mais en général les canoniers manquent à St-Domingue c'est une espèce d'hommes très nécessaire a y envoyer quant a la garnison et aux milices, on pense que si elles montent à 1500 hommes, elles seront a peu près suffisantes surtout si l'on y joint des negres esclaves comme il a deja été dit. La Rade est mauvaise, petite et ouverte ainsy les ennemis ne peuvent y rester longtems ny en grand nombre. Les côtes y sont bordées de Rochers escarpés jusqu'au port Marigot, ce qui leur a fait donner le nom de Côtes de fer: en sorte qu'elles sont aussy peu propres au débarquement qu'à établir une communication par terre aux ennemis, soit vers le Cap, soit vers St-Marc.

5 sic. 


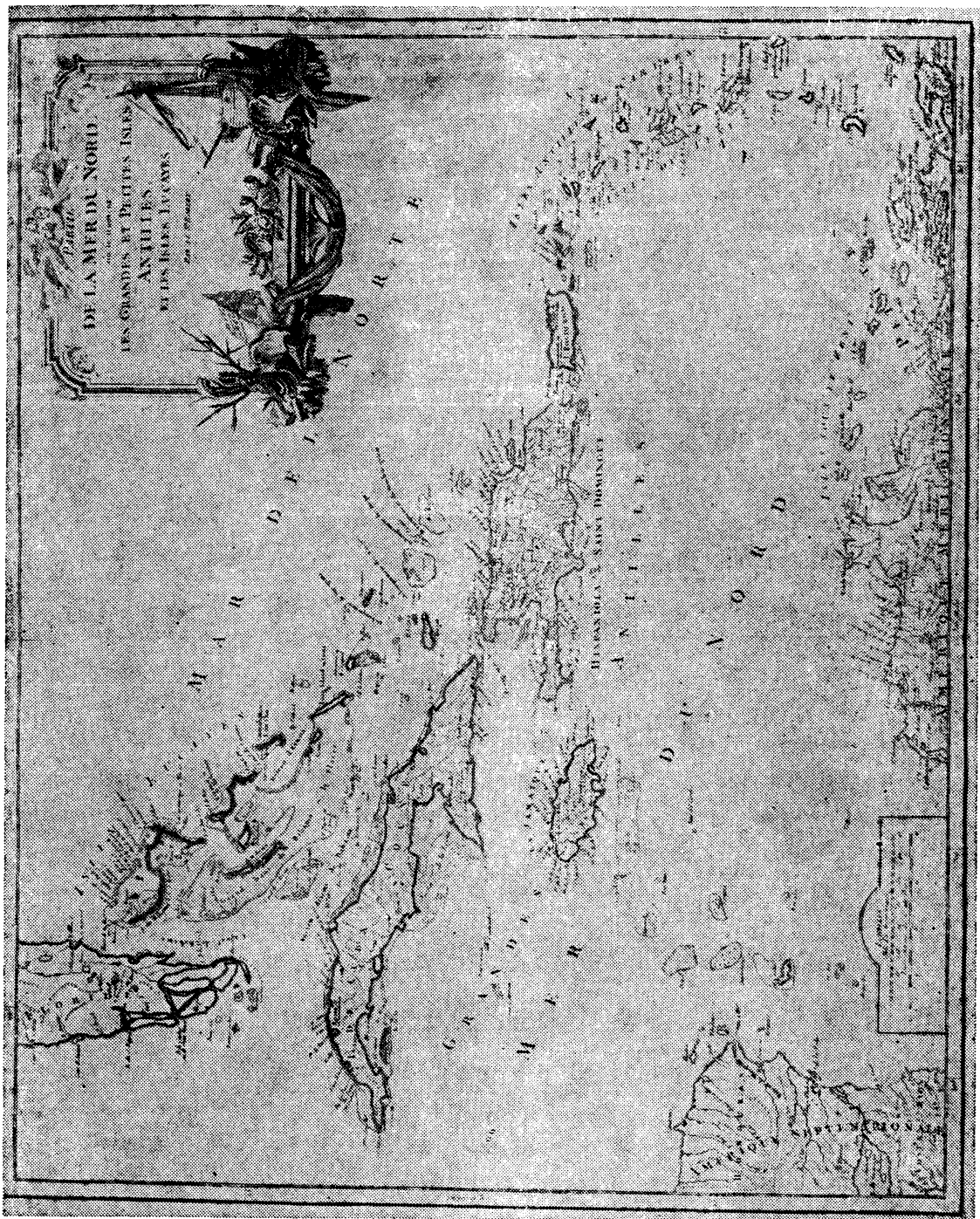

Carte de la "Partie de la Mer du Nord où se trouvent les Grandes et Petites Isles Antilles, et les Isles Lucayes, par le Sr Robert". 
Ce 3e établissement de la côte du nord de St-Domingue est d'une conservation beaucoup plus essentielle que le Port de Paix, parcequ'il couvre un pays riche, qui peut offrir un grand apas aux ennemis. Cependant il est presque entierement abandonné il est vray que sa Rade est foraine, et qu'il faut que les vaisseaux soient mouillés a terre pour trouver un fond convenable mais aussy les fortifications en sont si mediocres qu'elles ne peuvent resister au feu d'un escadre. D'ailleurs sans les attaquer directement il y a des endroits assés susceptibles de debarquement. Il est tres important d'y faire de bons retranchemens et de tenir les troupes reglées, les milices, et les negres toujours a portée de les garnir.

Le Port au Prince est le $4 \mathrm{e}$ port en consideration à StDomingue et le 1er de la partie de l'ouest. Il la doit cependant plus aux depenses qu'il a occasionnées jusqu'à présent qu'à sa valeur reelle. C'est un etablissement plus que mediocre, conseillé par M. de Vaudreuil ${ }^{6}$, et de peu ou de point d'utilité. $1^{\circ}$ parce que sa Rade est si étroite qu'elle ne contient qu'un petit nombre

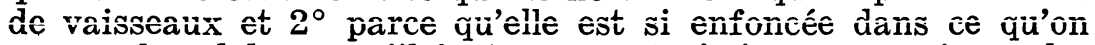
nomme le cul de sac qu'il faut souvent trois jours pour s'y rendre de la pointe de la Gonave à cause des calmes qui surprennent les vaisseaux dans ce détroit, ce qui la rend un azile inutile a ceux qui sont poursuivis et un poste desavantageux et mal situé pour ceux qui voudroient aller a la poursuite de l'ennemi que l'on sauroit en route pour la Jamaïque. Cependant comme les dépenses sont faites, et que le port au Prince est meme pris aujourdhuy pour le Chef Lieu de la colonie, par la residence du Gouverneur général que l'on y a fixée il convient de le fortifier autant que les circonstances peuvent le permettre pour cela il faut etablir une Batterie de canons et de mortiers sur une isle qui y forme deux especes de rades, et retrancher toute la partie de la coste de la droitte en allant au port au Prince ou il est facile de descendre au surplus il est necessaire que les troupes soient toujours prêtes a marcher au besoin; mais il est moins essentiel quelles soient aussy ramassées qu'ailleurs parceque les surprises et les attaques subites y sont moins a craindre.

Leogane est le 5e lieu qui mérite attention. C'étoit autrefois le principal azile de nos vaisseaux dans cette partie de l'isle mais ils étoient point à l'abry d'une insulte. La rade deffendue seulement et trop loin, pour un très petit fort est entierement

${ }^{6}$ Voir Guy Frégault, Le Grand Marquis. Pierre de Rigaud de Vaudreuil et la Louisiane (Montréal, 1952). 
ouverte et les ennemis peuvent y venir mouiller impunement il est difficile de comprendre par quel motif on s'étoit sy mal étably d'abord et surtout en batissant la ville a une demie lieue du debarquement sans la fortifier. Il falloit au moins qu'elle deffendit la Plage. A ce deffaut il est necessaire de la retrancher, et de tenir les troupes toujours a portée de s'y jetter, parce qu'il n'y a pas un endroit plus susceptible d'estre attaqué brusquement et qu'il couvre néanmoins une plaine d'une grande richesse dont la ruine causeroit une perte considerable.

Le Petit Goave, 6e etablissement français a St-Domingue n'est éloigné de Leogane que de 4 lieues c'est veritablement le seul ou la nature permette a l'art de faire une rade assurée, aussy propre a refugier nos vaisseaux, quand ils sont les plus foibles, qu'a mettre à portée de poursuivre et d'intercepter ceux des ennemis quand ils croisent sur le môle St-Nicolas, ou qu'ils traversent pour aller a la Jamaïque ou en revenir. Mr Larnage Gouverneur Général de St-Domingue le seul peut etre qui eut bien connu et bien administré jusque là cette colonie, avoit compris l'utilité du petit Goave; en consequence il sy retira au commencement de la guerre dernière et y fit à la hâte les meilleures deffenses quil put. On ne comprend point par quelle raison ce plan a été abandonné et lon doit la justice a $M$. du Bois de la Motte qui a gouverné a St-Domingue que jugeant du port au Prince comme on en doit penser il avoit formé le Projet de rétablir le chef Lieu de la partie de louest au petit Goave. Mais Mr de Vaudreil ${ }^{7}$ luy ayant succedé par un terme et trop longtems cet etablissement a été abandonné pour porter touttes les attentions au port au Prince qui étoit son ouvrage. C'est par ces changemens continuels depuis 40 ans que ce Boulevard tant cherché et pas encore trouvé, a couté peut être au de la de 40 millions. Il faut espérer qu'un gouverneur éclairé le fixera enfin; et que son choix tombera sur le petit Goave, que lon peut rendre imprenable facilement, et a assés peu de frais si l'on coupe dans ce pays là, et dans celuy-cy, sur tout ce qui concerne la Marine, les racines trop fécondes et trop multipliées des dépenses inutiles et des infidelités. La rade du petit Goave est bien fermée et son entrée est étroite. Elle peut être deffendue adroite par un fort considérable sur la pointe d'antoine à gauche par celuy qu'on placeroit sur une petite Isle de Sabre: et en face par des Batteries razantes qu'il faut établir au fond de la Rade. Tous ces feux peuvent se croiser en dedans sur les vaisseaux qui seroient parvenus à y pénétrer, malgré les feux

7 sic. 
extérieurs, et le fort a 4 Bastions qui avoit été élevé en terre dans la partie nommée accul les écraseroit egalement de son canon, et de ses bombes; ainsy il n'est pas vraysemblable qu'ils tentassent une entreprise ou ils échoueroient meme après un premier succès très douloureux, et qui leur auroit couté cher. D'ailleurs ce fort que l'on a appelé fort Royal, s'il étoit bien revetu, entourré d'un bon fossé, précédé d'un chemin couvert ne pourroit être pris que par un siège en règle; et le temps qu'il exigeroit, donneroit celuy de venir à son secours de tous cotés sans parler de l'intempérie du climat qui feroit perir plus d'hommes aux ennemis, que la deffense des assiégeans. En attendant que l'on puisse établir les choses solidement sur ce pied au petit Goave, il est de toute nécessité de relever en terre l'ancienne Batterie sur la pointe d'Antoine, d'en former une en face de l'Entrée et de se retrancher au fond de l'accul, si le fort Royal n'existe plus. Avec ses préparatifs à la hate, on assure que cet endroit sera encore mieux deffendu que ceux qui peuvent être fortifiés avec plus de soin.

On ne dira rien de Jaquemet ni de ( )$^{8}$ quoy que ces deux postes dussent meriter cependant que l'on s'en occupât. Il y en a tant d'autres plus importans a garder aujourd'huy qu'il ne faut considérer ceux là que comme des plages, praticables pour un débarquement et qu'il est par conséquent nécessaire de border de bons retranchemens avec la précaution d'avoir toujours des troupes à portée d'y estre conduites.

Le 7e et dernier etablissement de guerre, car les Cayes qui pouvoient et devoient cependant ( $)^{9}$ dont les Anglois s'emparerent avec tant de facilité pendant la guerre dernière. Il est au sud de St-Domingue et il offriroit une retraite très avantageuse a nos vaisseaux si la disposition de ses deffenses étoit mieux faite, mais on s'est borné a elever un fort si petit sur un amas de sable qui forme une Isle, que les éclats d'une bombe, qui tombe, au milieu sont poussés aux extremités. Ce fort cependant pouvoit être très utile s'il eut été mieux construit et deffendu par une Citadelle placée au-dessus de la ville, qui l'eut a la fois protégé pendant l'attaque, battu après la prise, et qui eut été un obstacle ensuite au débarquement. La nature du terrein adroitte, a gauche et en face de la rade est très propre a toutes les deffenses que lon voudra $\mathrm{y}$ faire, et ce parti est d'autant plus indispensable a prendre, que la rade de St-Louis est le seul azile de nos vaisseaux, en temps de guerre,

8 espace en blanc.

9 espace en blanc. 
dans toute la partie du Sud. La pointe nommée La Caye d'Orange doit être gardée par une Batterie de gros canons et de mortiers qui puissent tirer sur les vaisseaux ennemis longtems avant qu'ils se presentent à l'armée de la Rade. Il est facile de prendre des a présent cette précaution, en attendant que l'on puisse travailler avec solidité et de soutenir par des retranchemens garnis de canons les ruines du fort que les Anglois n'ont fait qu'ébranler en tentant de le démolir.

Jusqu'à présent on n'a parlé que des rades ou des mouillages que l'on a cru devoir occuper à St-Domingue; mais il en est resté plusieurs sans aucune deffense et ou, les ennemis peuvent néanmoins descendre sans effort, et s'établir de façon a en être chassés difficilement. Tels sont les endroits compris depuis le fort Dauphin jusqu'au Cap, et depuis, et depuis ${ }^{10}$ le Cap, jusqu'au port Marigot; il y faut nécessairement des retranchemens solides, et il convient de placer les troupes de façon qu'elles ayent entre'elles une communication assurée qui facilite leur réunion au point attaque. Il est important de prendre les mesmes précautions dans la partie de l'Ouest, mais surtout au Cap d'Anne Marie ${ }^{11}$ et a la baye des irois qui couvrent un etablissement qui pour être nouvellement formé n'en est pas moins considerable par les progrès rapides qu'il a faits, et qu'il doit a la bonté de ses terres.

Il est néanmoins livré jusqua present a la seule deffense de ses habitans; et ils ne sont pas encore en assés grand nombre pour resister a des forces serieuses. On a du pendant la guerre dernière le salut de cette contrée abandonnée a la discrétion des des ${ }^{10}$ Anglois qua l'interest qu'ils avoient a la conserver comme un debouché pour leurs nègres de contrebandes. Et c'est au profit que font les interlopes avec ces colons éloignés qu'il faut egallement attribuer les menagemens qu'ils ont eu pour quelques quartiers du Sud tout aussy exposés a leurs incursions et a leurs ravages mais cette politique de nos ennemis peut cesser par une differente façon de penser du Gouverneur de la Jamaïque ou par l'appas d'un gain présent toujours plus vif que celuy d'un gain a venir ainsy il est a propos d'etendre les vues de deffense sur tous les Lieux qui sont susceptibles d'attaque.

Mais il y en a un qui ouvrira toujours facilement la Rade $\mathrm{du}$ fort Dauphin parcequ'il ne depend point du Roy de le fortifier. C'est la Baye de Manceville qui appartient aux Espagnols,

10 répétition.

11 Dame Marie. 
qui ny ont formé aucun etablissement et dont les Anglois qui respectent peu les puissances quand leur canon ne leur en impose point, peuvent venir facilement et promptement par terre, pour prendre a revers le fort de la Bouque qui deffent l'entrée de la rade du fort Dauphin. Il ny avoit d'autre remede a cet inconvenient, que de faire de cette Bicoque une place de guerre alabry d'une insulte, et non de l'exposer comme elle lest par terre a etre enlevée par 500 hommes et meme sans echelles tant le mur qui la ferme est peu élevé.

On doit observer deux deffauts generaux dans les fortifications de nos colonies, sans parler des mauvais plans et de la mauvaise construction, le 1er est le peu détendue des places, le 2. leur foiblesse du coté de la terre ou elles sont même presque toujours commandées par quelque hauteur. Il semble que l'on n'ait jamais imaginé que l'on fut dans le cas d'estre pris a revers, et qu'on se soit cru bien en seurreté en se garantissant du costé de la Mer. Les Anglois a Minorque et dans toutes leurs colonies nous avoient cependant donne un exemple contraire qui étoit bon à suivre et qui ne rendroit pas inutile aujourd'hui la plus part de nos fortifications qu'il faut recommencer à la Paix, étendre, mieux placer, et mieux construire, si nous voulons résister a nos ennemis a l'exception du fort Royal de la Martinique, on ne connoist pas une place d'armes dans toutes nos colonies qui puisse meriter un siège et qui soit en état de servir de retraite au moins aux femmes, et aux Richesses de leurs habitans; on a pretendu que c'étoit par une défiance politique de leur fidelité; mais le danger n'est-il pas plus grand du coté de la facilité que l'on présente aux ennemis ? et d'ailleurs, n'est-ce pas risquer d'encourager des gens qui sont sans cesse exposés au pillage, et les tenter plustôt de s'y soustraire par une prompte soumission?

On ne peut le taire depuis trop longtems on les gouvernes 12 plustôt en ennemis pris a discretion quen citoyens et en sujets du Roy. On ne peut dissimuler non plus que vexés sans cesse, et toujours abandonnés, il ne soit peut être dangereux de les mettre a de plus longues epreuves et de ne point leur donner enfin des chefs qui y portent un esprit de douceur, de Justice et d'encouragement, qui ramenne les mecontens, contienne ceux qui sont ebranlés et fortifie ceux que le zèle soutient encore. En général le Roy na point de sujets plus fidelles plus ardens a se sacrifier aux choses qui concernent son service; mais il ne faut pas assés compter sur ces sentimens pour soufrir plus 
longtems qu'ils s'attirent par labus de l'autorité surtout avec des peuples qui nont guere d'autre frein que leurs devoirs qui seroient soutenus dans leur désobéissance, et qui y trouveroient la fin des Injustices qu'ils essuyent et de la disette qu'ils éprouvent.

Il y a peu de jours quon a pû voir dans les gazettes de Londres que lon cherche a echauffer dans l'esprit du Gouvernement le desir qu'il a certainement dejà de faire la conquette de la plus riche de nos colonies. M. Pitt n'étant que simple citoyen n'a jamais en d'autre plan, et il n'a désiré de parvenir au Ministere, que pour avoir les moyens de l'executer. On lit dans ces gazettes que les habitans de St-Domingue qui ont été pris sur nos vaisseaux marchands sont les premiers a encourager nos ennemis a diriger leurs forces sur cette colonie, au lieu de les tourner contre des objets aussy peu considerables que ceux qui les ont occupés jusqu'à present. On peut croire sur cela que leurs papiers publics outrent la chose; mais il n'en est pas moins vray que le mécontentement est si général qu'il est possible qu'il ait eclatté en de pareils discours et qu'il eut peut etre des effets facheux. Les Anglois trop instruits qu'il existe peuvent tenter avec succès pendant cette guerre le moyen qu'ils voulurent mettre en usage pendant la dernière pour corrompre la fidelité de la pluspart des habitans de St-Domingue. Ce moyen étoit de laisser à chaque propriétaire présent son patrimoine, et de donner ceux des absents à leurs représentans sils refusoient de se joindre aux troupes reglées pour empecher la conquete du pays. Il eut été a craindre qu'un pareil objet de séduction n'eut opéré l'effet que les ennemis en attendoient, et il seroit dangereux qu'ils ne l'operat encore aujourd'huy. Il ne faut pas perdre de vue que la moitié des colons régit les biens de l'autre moitié absente. L'auteur du mémoire qui entretenoit une intelligence en Angleterre avec la permission du Roy pendant la guerre dernière fut informé a tems de ce dessein, ainsy que l'entreprise méditée sur le fort de St-Louis. Il en rendit compte à $\mathrm{Mr}$ de Maurepas qui eut lieu six mois après de juger de la sollidité de l'avis. Si ce projet ne fut pas executé dans toute son etendue, c'est que les Anglois occupés chez eux mêmes ne purent réunir toutes les forces qu'il exigeoit; mais aujourd'huy rien ne paroist les arrester.

Il n'est que trop vraisemblable que la garantie de l'Espagne ne sera plus un obstacle a leurs vues sur nos possessions de l'Amérique meridionale, depuis que notre marine éteinte est hors d'etat de seconder celle de cette puissance. En vain recla- 
mera-t-elle les traittés; l'Angleterre fera peu d'attention a des menaces qui ne peuvent être suivies d'effets redoutables: car il ne faut pas croire que la seule marine Espagnole dont les operations d'ailleurs sont encore plus lentes que les notres soit assez forte pour sopposer aux entreprises de la marine angloise on croit que l'Espagne a fait une faute d'autant plus grande de ne se pas declarer dès le début de cette guerre qu'elle a certainement été, autant entreprise contr'elle que contre la france, quoiqu'en apparence moins directement. Elle ne doit jamais oublier que l'ambition de l'Angleterre, outre son projet de commerce universel, et d'abaissement de toutes les puissances maritimes attaquées separément est de former a ses depens un Etablissement a la mer de Sud qui luy ouvre les trésors du nouveau monde. Ce fut l'unique motif de la guerre qu'elle fit aux Espagnols en 1740 et des efforts qu'elle tenta sur Port Bello, et Cartagene garants de ses possessions dans cette partie de l'Amerique, comme elle l'est des notres, nous n'attendimes point qu'elle fut épuisée pour la secourir et le Roy fit armer 32 vaisseaux de touttes grandeurs cest a dire tout ce qui composoit

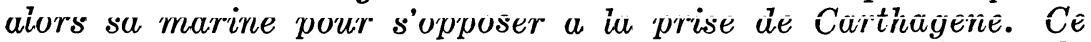
secours en imposa aux Anglois; tout médiocre qu'il étoit, par la foiblesse des vaisseaux, et par le défaut des vivres qui les obligea de revenir dans nos ports trois mois trop tôt, cependant ils ne contribuerent pas moins a la levée d'un siège entrepris seulement après leur retour, et dans les mois Brillans de l'année qui firent perir plus de 10 mille hommes aux Ennemis.

Quand ils ont commencé cette guerre; outre le projet de détruire notre commerce trop florissant et d'empecher le retablissement de notre marine, ils ont egalement eu celuy de parvenir a attaquer en suitte plus facilement les Espagnols après nous avoir mis hors d'etat de leur porter aucuns secours par la disgrace du Mis de La Encenada qu'ils ont occasionnée, et le choix des Ministres, que leurs intrigues luy ont donné pour successeurs, ils se sont assurés de linaction de l'Espagne pendant qu'ils nous affoibliroient et des moyens d'envahir ensuite plus aisement ses possessions. Notre exactitude a remplir nos engagemens leur enseigne la route differente qu'ils devoient tenir, pour parvenir a la même fin. Si le Ministre espagnol eut été moins anglois, il étoit impossible qu'il ne penetrat point ce projet caché. D'ailleurs celuy de la domination de la mer a été trop affiché par les Anglois pour que toutes les puissances maritimes ne s'alliassent point afin de le faire echouer. Si l'equilibre de la balance des forces de terre de l'Europe est un point réel et politique qui en doive occuper les puissances pourquoy la balance 
des forces maritimes n'auroit-elle pas besoin des memes contrepoids? 13 Cependant on a vu avec une espece d'indifférence les marines francoises et Angloises aux prises comme si la ruine de la 1re n'entrainoit pas celle de la Marine et du commerce des autres Nations et que sa cause ne fut pas la cause commune.

Il est vray que la faute énorme que nous avons faite de refuser longtems l'entrée des ports de nos colonies aux vaisseaux des puissances neutres a pu et du ralentir leur interest, et quelle nous a privé d'alliés necessaires, soit que les Anglois respectassent ou non leurs Pavillons. C'est une faute que l'on voudroit reparer aujourd'huy; mais il est a craindre que l'on ne puisse y parvenir. Cependant la ressource des neutres seroit la plus sure pour approvisionner nos colonies depourvues de vivres et de munitions de guerre; et il faut tenter ce moyen quoyqu'il ne soit que trop a presumer que les Anglois s'empareront de leurs vaisseaux. Ils seront fondés, en arretant tout ce qui sera prohibé, et les munitions de guerre passeront pour telles. Ils arresteront egalement les vaisseaux qui n'en seront point chargés et cela sans rompre avec les puissances dont ils porteront les couleurs, parce qu'après lexamen des raisons respectives des vaisseaux pris, ils relacheront ces derniers, satisferont ainsy leurs maitres, et nen feront pas moins échouer le ravitaillement et le secours de nos colonies.

Il n'y a d'autre expedient pour encourager les neutres a s'en charger, malgré ce danger trop apparent, que d'assurer l'atterage de nos isles, par des escadres qui y croisent, et surtout beaucoup de fregate qui les purge de la quantité de Corcaires ${ }^{14}$ ennemis qui les infectent: ce sont eux qui ont fait presque toutes les prises qui ont rebuté les hollandois; et il est surprenant que lon ait vû jusqu'à present ce mal auquel il etoit si facile de remedier, avec tant de tranquillité.

Si l'armement de l'armée ${ }^{15}$ dernière de Onze vaisseaux de guerre et de plusieurs fregattes eut été fait dans l'océan, au lieu de la Mediterranée ou si cette forte escadre se fut combinée a Toulon pour forcer le detroit de Gibraltar, et que tous les vaisseaux armés a Brest fussent partis ensemble il est certain que Louisbourg eut été sauvé et que nos autres colonies ne seroient pas exposées au danger pressant qui les menace.

\footnotetext{
${ }^{13}$ Le thème de l'équilibre entre les forces navales des Etats maritimes figure dans un document de Maurepas, cité par Roland Lamontagne, Aperçu structural du Canada au XVIII e siècle (Montréal, 1964), 66-67.

14 sic.

15 On devrait lire: année au lieu de armée.
} 
On peut aprecier icy au juste la quantité de munition de guerre qu'il faudroit envoyer a St-Domingue ce calcul depend de celuy de la poudre, des canons, des boulets, des affuts, des mortiers et des bombes qui y sont actuellement. Mais il est facile a faire d'après la numeration des differents ports dont on vient de parler, et qu'il est essentiel de munir; quant aux troupes, il est certain que l'on ne scauroit trop y en envoyer, mais il faut en même tems compter sur les vivres necessaires pour les nourrir. C'est encore un article que l'on ne doit pas craindre de porter trop loin.

Le besoin actuel de comestible est aussy grand a St-Domingue, que le debouché et la consommation en sont assurés; ces trois points si essentiels, munitions de guerre, hommes et vivres sont si pressants qu'il ne faut même pas sen raporter uniquement aux vaisseaux neutres et qu'il faut en envoyer des nationnaux: mais toujours dans la suposition que l'on nétoyera précédemment les cotes de St-Domingue.

Si dès le commencement de la guerre, le commerce de cette

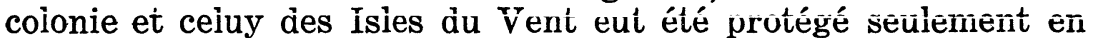
facilitant l'accès de leurs ports, il est constant que le défaut de credit ne seroit point fait sentir en France, et que la confiance ne s'y seroit pas perdue. L'exemple de la guerre dernière en est une preuve. Nous avions une marine bien moins considerable quau debut de celle-cy, cependant malgré toutes nos pertes sur mer, il n'y eut point d'armée qui ne fit entrer dans le Royaume par le commerce depuis 100 jusqu'a 130 Millions d'argent etranger. Cette ressource remplaça celuy qui en sortoit pour les subsides, et facilita toujours les emprunts, le contraire est arrivé aujourd'huy par l'unique abandon de ce meme commerce, qui n'etant point soutenu n'a servi qu'a enrichir nos ennemis, ruiner nos classes et allarmer tellement ceux qui pouvoient prêter de l'argent au Roy, que touttes les Bourses ont été fermées, et qu'il a fallu recourir a des impôts insufisants pour subvenir aux frais de la guerre. Les speculateurs voyans sans cesse les finances du Royaume exportées à l'etranger et jamais celles de l'etranger importées chez nous par la voye du commerce ont craint que les notres ne sepuissassent entierement avant de pouvoir être remplacées. Et cette frayeur est cause de la situation embarassante ou nous sommes, que tout le monde ne sent que trop et que peu de gens savent a quoy attribuer tant nos colonies et notre commerce sont mal connus.

On n'entre icy dans aucun détail particulier des Isles du Vent parce qu'il n'y auroit a peu près que les mêmes choses 
a en dire, tant par raport a la seureté de leurs ports qua leurs garnisons, leur aprovisionnement et leurs munitions. Il suffit d'examiner leur état present comme on vient d'exposer celuy de St-Domingue pour connoistre le point d'ou il faut partir a leur égard. Il s'agit particulierement de la Guadeloupe, la Grenade, Marie Galande, Ste-Lucie, La Dominique et St-Vincent; car on peut assés considerer La Martinique comme une colonie hors d'insulte, et meme a l'abri des entreprises les plus serieuses de l'Angleterre tant par la nature de son terrain que par la Citadelle du fort Royal, le nombre et la valeur de ses habitans. Cette Isle qui na pas plus de 40 lieues de circonférence a peut être dans ses colonies plus de 12 mille deffenseurs ainsy elle ne doit être l'objet des soins du Gouvernement dans la circonstance actuelle qu'en ce qui concerne les munitions de guerre et de bouche.

Au reste on seroit d'avis et l'on croit que la France en a un droit fondé sur la garantie de l'Espagne de s'assurer des dispositions de cette couronne dans le cas ou les Anglois attaqueroit St-Domingue, le secours le plus certain, le plus prompt et le moins exposé à manquer, seroit celuy des troupes et des habitans espagnols qui partagent cette isle avec nous, mais il faudroit que les ordres leur en fussent donnés d'avance de Madrid, que leurs dispositions fussent faites, et qu'il y eut même des corps déjà placés sur nos frontières pour être à portée de nous joindre a laproche des ennemis. Il ne seroit plus tems de reclamer la garantie dans cette circonstance, ny meme d'en attendre les effets, quand même l'Espagne voudroit agir en nôtre faveur et remplir leurs traittés.

Grâce à une subvention accordée en 1965 par le Ministère des Affaires culturelles du Québec, les précisions touchant l'administration de Maurepas résultent d'une recherche en cours dans les Fonds Maurepas, comme suite à l'ouvrage intitulé: L'Atlantique jusqu'au temps de Maurepas (Les Presses de l'Université de Montréal, 1965). 